\title{
Bases epistemológicas y operativas de la didáctica del resumen documental: un enfoque basado en la competencia resumidora
}

Mónica Izquierdo Alonso

Facultad de Documentación. Universidad de Alcalá Madrid, España

Carmen Sánchez Domínguez

Facultad de Documentación. Universidad de Alcalá Madrid, España

Se destaca la necesidad de incluir una dimensión pedagógica dentro de las investigaciones de tratamiento documental de contenido, centrando la atención en el enfoque didáctico del resumen. Se abre así una nueva vía de análisis, desde la didáctica del resumen documental y la adquisición de la competencia resumidora. Se definen y caracterizan los principios básicos de esta didáctica, fundamentados en la noción de competencia y se describe un modelo holístico para aquella, describiendo sus componentes y funcionamiento.

Palabras clave: Didáctica del resumen documental; Enseñanza-aprendizaje del resumen; Competencia resumidora; Diseño instructivo con competencias (modelo de proceso); Diseño curricular orientado hacia competencias (modelo de objetivos).

\section{Epistemological and methodological basis of documentary abstracting didactics: an abstracting competence approach}

It stresses the need to include a pedagogical perspective into research documentary content treatment, focusing on didactical approach of the abstract studies. A new field research is open from the abstracting competence and its acquisition. The theoretical framework focused on 
competencies is defined and the implications for the career instructive design are outlined. Moreover, a holistic and dynamic model for abstracting competence is described. This competency model is reviewed from a distinction between competence (the underlying system of knowledges, skills, attitudes, etc.) and performance (abstracting). Moreover, abstracting competence is made up of a system of sub-competences that are strongly interrelated, hierarchically sequenced relations and subject to variations in context. This abstracting competence is actualised in different ways in different situations.

Keywords: Didactical documentary abstract; Teaching and learning of abstracting; Abstracting competences internal dimensions; Competencies based instructive design (objectives model); Abstracting curriculum design with competences (process model).

Recebido em 28.10.2009 Aceito em 18.10.2010

\section{Introducción}

Uno de los conceptos clave que ha impulsado una transformación en los estudios de resumen, en especial en su aprendizaje, ha sido el de proceso. Proveniente de la psicología cognitiva, y con interesantes desarrollos en campos afines como el de la enseñanza de la lectura y la composición escrita (FLOWER; HAYES, 1980; COLLINS; GENTNER, 1980; WINOGRAD, 1984; JOHNSON, 1989; GRAVE; KAPLAN, 1996; CASSSANY 1998; SCARDAMALIA; BEREITER, 1987; 1992, etc.), supone una nueva mirada hacia el resumen documental, condiserándolo desde su dimensión de actividad ${ }^{1}$. De este modo, se incorporan nuevas nociones a este producto documental como las de subproceso, actuación, destreza o competencia, así como toda una rica temática de investigación asociada: planificación y secuenciación de procesos, adquisición y uso de estrategias, estudio de desempeños eficientes, identificación y resolución de problemas como núcleos de procesamiento de tareas, etc., que enriquecen la investigación del objeto de estudio en nuestra disciplina. Este tipo de enfoque aplicado al resumen documental, con orientaciones cognitivistas o sociocognitivistas, centra su atención en el análisis de de la práctica resumidora y en el sujeto/contexto como agente(s) activo(s) del proceso. Se abren así diversos frentes de investigación orientados hacia: a) los componentes y naturaleza de las distintas etapas de la actividad y los procesos sociocognitivos subyacentes, b) la calidad de los procesos y

\footnotetext{
1 Para un desarrollo más detallado de los modelos en el resumen documental Vid. Izquierdo Alonso y Moreno Fernández (2010), Izquierdo Alonso (2009) o Pinto (2001).
} 
su evaluación, c) las diferencias entre resumidores expertos y noveles, o d) los procesos de enseñanza/aprendizaje (didáctica resumidora). Por otra parte, el modelo del Espacio Europeo de Educación Superior (EEES) conlleva una readaptación de los diseños curriculares, enfocándolos hacia el desarrollo de competencias y a la existencia de un conocimiento experto, como valor máximo en la adquisición de competencias. Todo ello supone un punto de inflexión para nuestras investigaciones sobre resumen, justificando la necesidad de principios y criterios didácticos más sistemáticos y operativos, desde la observación de situaciones y contextos reales de aprendizaje, la definición de diseños curriculares adecuados, la categorización de errores, la identificación de procesos específicos de intervención-supervisión para el alumnado así como el estudio de estrategias desde la identificación de problemas, para la mejora de la actividad en el aula. Este análisis de la práctica resumidora en toda su complejidad supone un punto de partida para la elaboración de una teoría de la acción didáctica en el resumen documental (FIG. 1).

La experiencia docente en el resumen documental nos ha llevado a defender una perspectiva didáctica fundamentada en el interaccionismo sociodiscursivo, en la que el alumno ha de conjugar una serie de elementos interdependientes y situados contextualmente tales como: definición de los objetivos de la tarea, planificación de procesos, desarrollo de distintos tipos de destrezas, resolución de problemas en las distintas fases de la actividad resumidora así como la identificación y superación de errores para mejorar la práctica resumidora. La parametrización de errores desde un enfoque semiótico y comunicativo constituirá un punto vital para configurar el diseño instructivo y para que el propio alumno o profesional detecte los puntos fuertes y débiles de su acto resumidor.

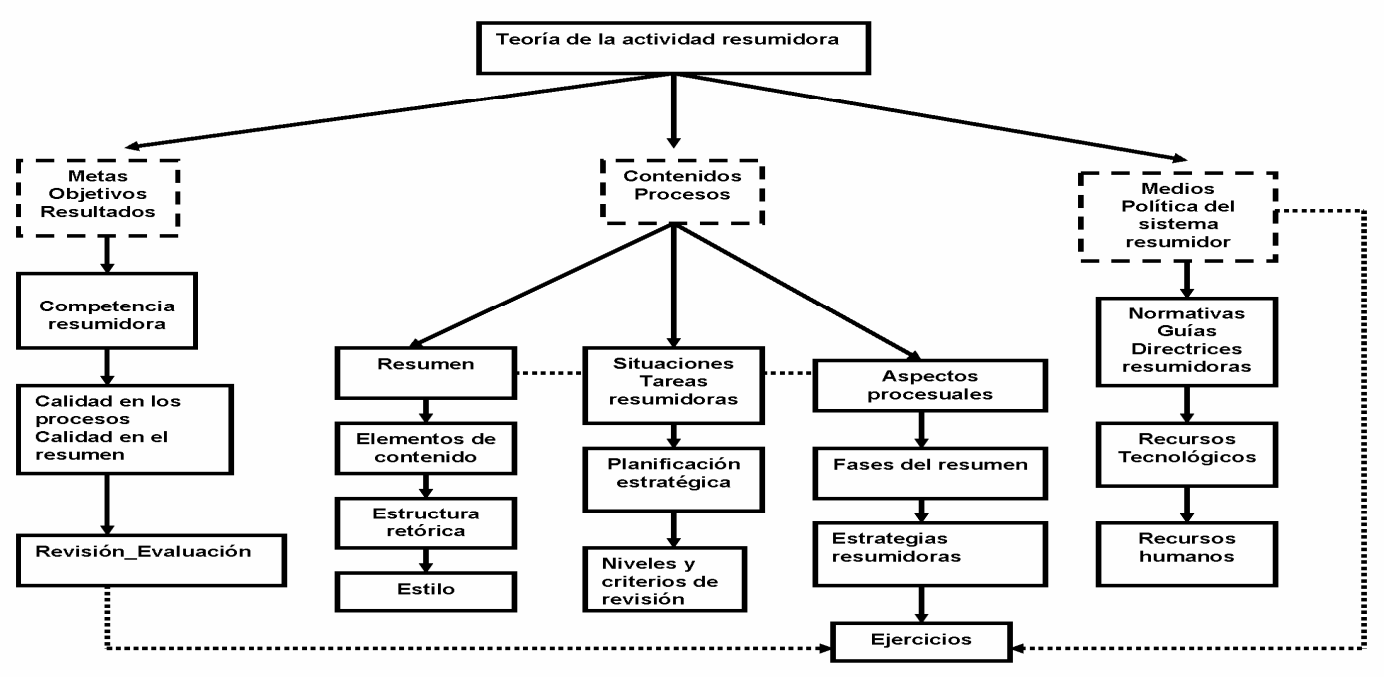

FIGURA 1 - Componentes de la teoría de la acción resumidora

Fonte: Elaboración propia. 


\section{Didáctica del resumen documental: la emergencia de un área específica de conocimiento en los planes formativos de biblioteconomía y documentación}

La reorganización de los estudios de educación superior ha traído consigo una remodelación de los diseños curriculares y una renovación de los procedimientos de enseñanza-aprendizaje. Ello conlleva una necesaria reflexión sobre los principios didácticos generales, adaptados a las necesidades concretas de cada disciplina y subdisciplina. En el caso de la Semiótica Documental, este hecho supone incluir nuevos espacios didácticos, desde la dimensión docente e investigadora, para las distintas operaciones del tratamiento documental de contenido (TDC): clasificación, indización y resumen. Esta asunción lleva implícita un modelo didáctico general, común a todas las técnicas de representación documental, así como didácticas más específicas para cada una de aquellas. De este modo, definiremos la didáctica de TDC como una dimensión teóricopráctica e interdisciplinar, con espacios transversales y dinámicos, integrados por las Ciencias de la Educación y la propia Semiótica Documental. Su objetivo sería desarrollar y mejorar la macro-competencia de organización y representación documental. Por otra parte, habría de ofrecer una descripción y explicación del funcionamiento de los procesos y productos del TDC, de modo que ayude al docente a planificar y supervisar su enseñanza y, al estudiante o profesional, a reflexionar sobre las limitaciones y potencialidades que le plantea la práctica del TDC. Delimitado el marco general, centraremos nuestra atención en el escenario didáctico del resumen documental.

A pesar de que existen vestigios de la técnica resumidora ya en la antigua Mesopotamia y de que desde 1970 se revalorizan las técnicas resumidoras con un marcado carácter prescriptivo, influenciadas por las investigaciones en técnicas de composición escrita, la investigación pedagógica no se incorporará a los estudios de resumen hasta inicios de los años $90^{2}$. Y ello desde consideraciones generales sobre los procesos de enseñanza-aprendizaje del análisis documental de contenido ${ }^{3}$. Ésta aproximación didáctica exige una reorientación metodológica y una reconducción de los objetivos y técnicas didácticas.

\footnotetext{
2 Para un estudio más detallado de la evolución del resumen documental desde la comparación de distintos modelos y enfoques Vid. Izquierdo y Moreno (2010) e Izquierdo Alonso (2009).

${ }^{3}$ Contamos con una importante herencia teórica que nos permite conocer el funcionamiento del resumen y los procesos de la actividad resumidora. Hemos de establecer, no obstante, una distinción entre revisión teórica, reflexión teórica sobre la práctica, y los planteamientos pedagógicos para adquirir dicha práctica (didáctica). Así, entre las investigaciones sobre resumen documental encontramos: a) estudios teóricos generales, que cubren los principios básicos del resumen y las fases de su elaboración; b) monografías centradas en aspectos puntuales como: el procesamiento textual y/o psicocognitivo en el resumen, la evaluación y calidad, la automatización del resumen documental, etc.; y c) manuales didácticos, enfocados hacia la práctica resumidora, con selección de casos comentados y propuesta de soluciones. Sin embargo, existe un vacío generalizado a la hora de afrontar estas propuestas y aplicarlas en un contexto real. En este sentido, las orientaciones didácticas irían enfocadas hacia: a) a formulación de competencias y objetivos pedagógicos, b) la definición de criterios metodológicos explícitos, c) la identificación de los problemas-errores y dificultades del aprendizaje en la actividad resumidora, etc. Para un estudio más detenido del espacio didáctico en el resumen documental, así como de la evolución y desarrollo de sus propuestas didácticas Vid. Izquierdo y Moreno (2009).
} 
Concebiremos el concepto de didáctica general del resumen como aquellos principios y métodos básicos de aproximación al concepto y actividad general resumidora que permiten al alumno sumergirse e iniciarse en los procesos específicos de condensación documental. Este primer nivel general le ayudará a enfrentarse, posteriormente, al contexto de los resúmenes especializados que requieren de técnicas y estrategias más elaboradas por la propia complejidad de los contextos, códigos y discursos. Así pues, el adjetivo especializado puede atender a criterios distintos:

Al dominio temático (así, en la esfera científica las distintas áreas como ciencias humanas, sociales, sanitarias, tecnológicas, etc.)

A los ámbitos discursivos o esferas sociales de actividad (científica, profesional, institucional) y

A los canales o modos tras los que se emiten los mensajes que determinan la naturaleza semiótica del contenido y las técnicas específicas para afrontar el resumen de estos códigos (resumen de documentos textuales, icónicos, audiovisuales, recursos electrónicos, etc.).

Esta dicotomía didáctica, resumen general y especializado, nos abre hacia una nueva realidad profesional: a) la del resumidor especializado en diversas esferas de actividad (científica, económica, jurídicoadministrativa, técnica, etc.) y b) la de un especialista semiótico en resúmenes icónicos, audiovisuales, recursos electrónicos, etc. De este modo, situamos la figura del resumidor en los distintos dominios de la actividad profesional y en todos aquellos ámbitos relacionados con la generación, comunicación y control normalizado de la información.

Contextualizado el espacio didáctico del resumen documental, definiremos la enseñanza/aprendizaje del resumen documental como la generación de aquellos contextos adecuados para que se produzca un aprendizaje efectivo del proceso resumidor, dentro de una situación comunicativa documental. Esta enseñanza ha de profundizar en diferentes aspectos de la práctica resumidora; desde la formulación de estrategias para la solución de problemas específicos en distintas situaciones de aprendizaje, según la adquisición de diferentes niveles de competencia resumidora (desde el novel hasta el experto).

Su objetivo final será el logro de una adecuada competencia resumidora que permita:

a) conocer los principios fundamentales del resumen, así como las técnicas básicas necesarias para aplicarlas a la elaboración y evaluación de resúmenes documentales, según el contexto de la actividad y el proyecto requerido.

b) dominar las herramientas tecnológicas específicas para la gestión de resúmenes en distintos contextos de especialidad, desde un enfoque estratégico de la actividad resumidora. 
c) adaptarse a los constantes cambios tecnológicos que inciden en los canales, códigos y géneros de los materiales a resumir, y en la propia naturaleza del de resumen documental.

d) asumir un sentido ético ante la representación documental y una responsabilidad con el rol del ejercicio de la profesión.

\section{Los componentes de la propuesta didáctica}

Una vez definido el objeto de la didáctica específica del resumen documental como el espacio de interacción entre las prácticas pedagógicas y los procesos de aprendizaje, analizaremos cada uno de los componentes y principios del modelo didáctico: competencias, objetivos y estrategias didácticas. Nos detendremos especialmente en el concepto de competencia resumidora, pues sobre esta noción se ha de erigir el enfoque didáctico. No es nuestra intención profundizar en todos los aspectos del diseño curricular, que sin duda merecerían un estudio independiente que excede los límites que nos hemos marcado en este trabajo ${ }^{4}$.

\section{1 La competencia resumidora}

Es éste uno de los conceptos clave de la didáctica del resumen. Su adquisición y consolidación constituye el objetivo final de la formación del resumidor profesional. La definiremos y describiremos un modelo propio para su funcionamiento, desde el análisis de sus componentes y las interrelaciones entre éstos.

\subsubsection{Hacia una definición de competencia resumidora documental}

La competencia resumidora es un término relativamente nuevo, si tenemos en cuenta que empieza a ser utilizado por los autores que escriben sobre nuestra disciplina a mediados de la década de 1990. Y ello, ante un creciente interés por el estudio del proceso resumidor, bien al calor de la perspectivas cognitivistas, que enfatizaban conceptos como los de estrategia o destrezas en el procesamiento de la información, o bien desde una renovación de los enfoques pedagógicos. Sin embargo, y aunque se alude al concepto en términos de técnica y habilidad resumidora, en la mayoría de las ocasiones no existen definiciones explícitas. En general, se hace referencia a la noción de competencia

\footnotetext{
4 Un análisis completo de los componentes del proceso de enseñanza aprendizaje incluiría: definición de objetivos de aprendizaje; formulación y secuenciación de contenidos; diseño de metodologías y estrategias de enseñanza-aprendizaje adecuadas; elaboración de materiales didácticos y planificación de actividades significativas; formulación de criterios y metodologías de evaluación, orientadas hacia la adquisición de competencias; detección de problemas en el desempeño de la actividad resumidora; definición de modelos de intervención y supervisión docente, descripción de criterios y parámetros de evaluación desde la categorización y tipificación de errores, etc.
} 
resumidora con una definición genérica: como habilidad para saber resumir, dentro de una caracterización general del proceso resumidor y la evaluación de la actividad resumidora. De este modo, es una noción implícita al acto resumidor del profesional de la información o del autor que realiza el resumen.

Paralelamente a la distinción chomskyana ${ }^{5}$ entre competencia (performance) y actuación lingüística distinguiremos los conceptos de competencia (sistema subyacente de conocimientos y habilidades) y actuación (acto resumidor). Así, concebiremos la competencia resumidora como un conjunto de conocimientos, procedimientos, actitudes y capacidades que una persona posee y son necesarias para:

afrontar de forma efectiva las tareas que requiere la actividad resumidora con el nivel y calidad de desarrollo requeridos.

resolver los problemas de un proyecto resumidor con iniciativa, autonomía y resolución.

adaptarse al entorno sociolaboral del contexto resumidor y del mercado global de la información desde un compromiso ético.

Es una suma integral de atributos y valores aplicables a distintas dimensiones: semiótico-discursiva, documental, gramatical, sociocultural, táctico-estratégica, procedimental, cultura de la profesión, etc.

El estudio de esta competencia resulta especialmente relevante desde un punto de vista pedagógico, ya que nos ayuda a establecer precisamente cuáles son los objetivos generales y específicos de la formación del resumidor $y$, al mismo tiempo, nos permite medir el desempeño y la cualificación resumidora. Por otra parte, el análisis de esta competencia es un aspecto complejo y heterogéneo, dada la multiplicidad de factores que entran en su naturaleza y medición. Constituye, pues, otra de las aproximaciones clave al concepto de resumen documental y de la actividad de resumir. Sintetizamos en el la siguiente figura los componentes, que ha nuestro juicio, han de formar parte de esa competencia resumidora:

\footnotetext{
${ }^{5}$ Vid. Chomsky, 1965.
} 


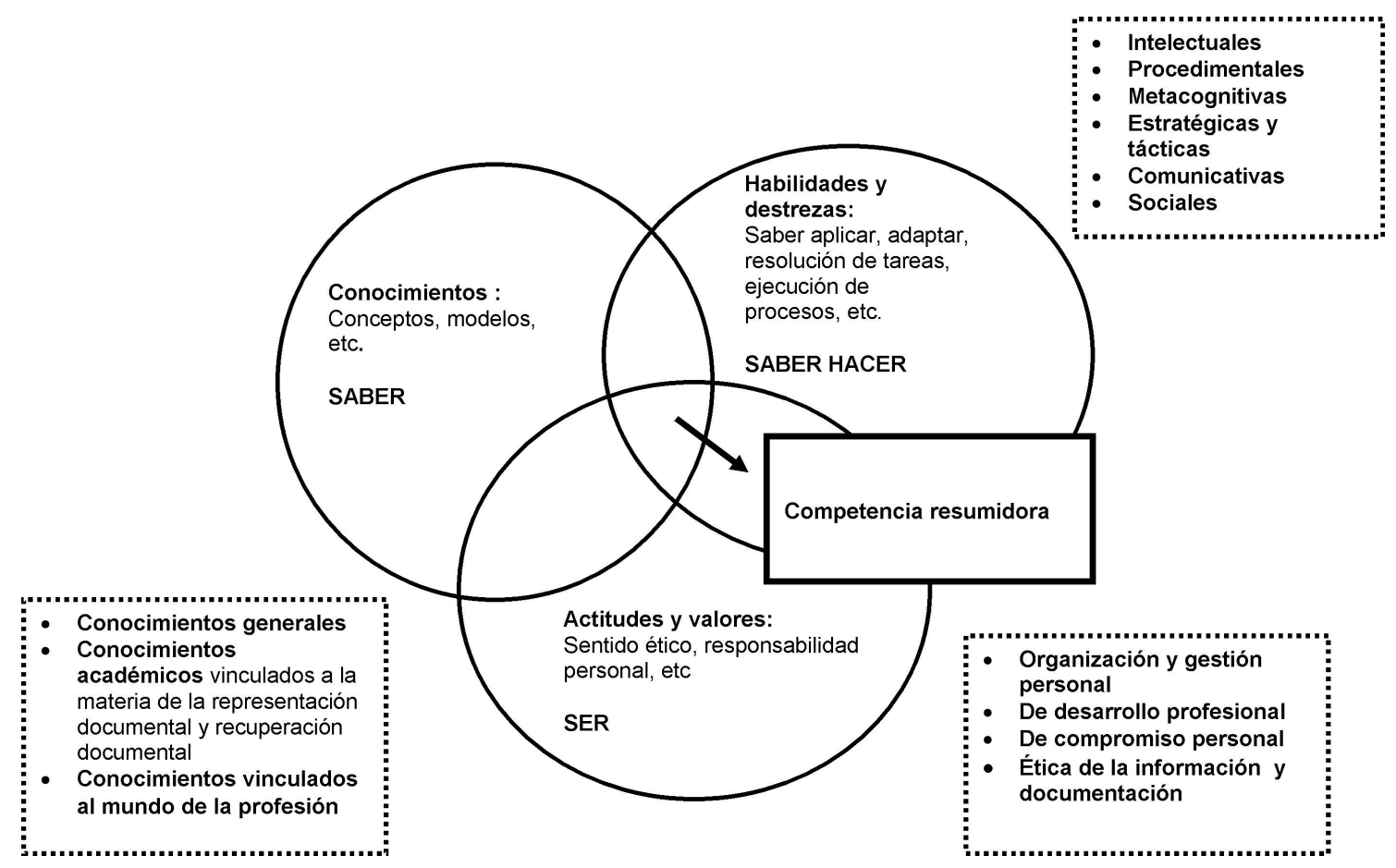

FIGURA 2 - Las dimensiones de la competencia resumidora Fonte: Elaboración propia.

La naturaleza de la competencia resumidora y de sus componentes ha sido tratada, de un modo indirecto, en el libro blanco en Información y Documentación (2004) así como en la relación de eurocompetencias, presentada por distintas asociaciones profesionales del mundo de la Biblioteconomía y Documentación (TEJADA; MEYRIAT, 2003; ECIA, 2004) en un intento de aunar intereses profesionales y académicos para definir los perfiles profesionales y diseñar un curriculum académico orientado hacia las competencias ${ }^{6}$. De un modo más específico, otros autores han integrado distintos catálogos de competencias en materia de representación documental (IZQUIERDO ALONSO, 2000, p. 163; PINTO et al., 2005, p. 31; GARCÍA MARCO et al., 2006; IZQUIERDO; MORENO, 2009).

\subsubsection{Características de nuestro modelo integrado de competencia resumidora}

Hemos de señalar las dificultades que encierra elaborar un modelo de competencia resumidora, dada la dimensionalidad del propio término competencia y la complejidad del acto resumidor. Dicho modelo ha de ser validado durante el proceso de aprendizaje del resumen, en sus distintas fases, valorando el desarrollo e interacción de cada una de las

6 Cfr. Moreiro y Tejada, 2004; Tejada Artigas et al. 2006. 
competencias y subcompetencias, y atendiendo a los diversos niveles o estadios en el aprendizaje de esta técnica de representación documental.

Las características generales que definen el modelo son las siguientes:

Interrelación de competencias locales para comprender la competencia resumidora global como un sistema complejo de conocimiento.

Incorporación a las competencias resumidoras de un enfoque sociocomunicativo y discursivo.

Integración de competencias cognitivas, procedimentales, metacognitivas y de desarrollo y compromiso profesional.

Inclusión de un componente profesional y cultural sobre toda la labor de representación documental [competencia y cultura profesional].

Definida la naturaleza de nuestro modelo dinámico de competencia resumidora haremos un desglose de competencias y subcompetencias. Al mismo tiempo, señalaremos que la competencia resumidora consiste tanto en el conocimiento de las reglas y convenciones del resumen como en la capacidad para ejecutar todas las subcompetencias en contextos reales y ante distintas situaciones resumidoras.

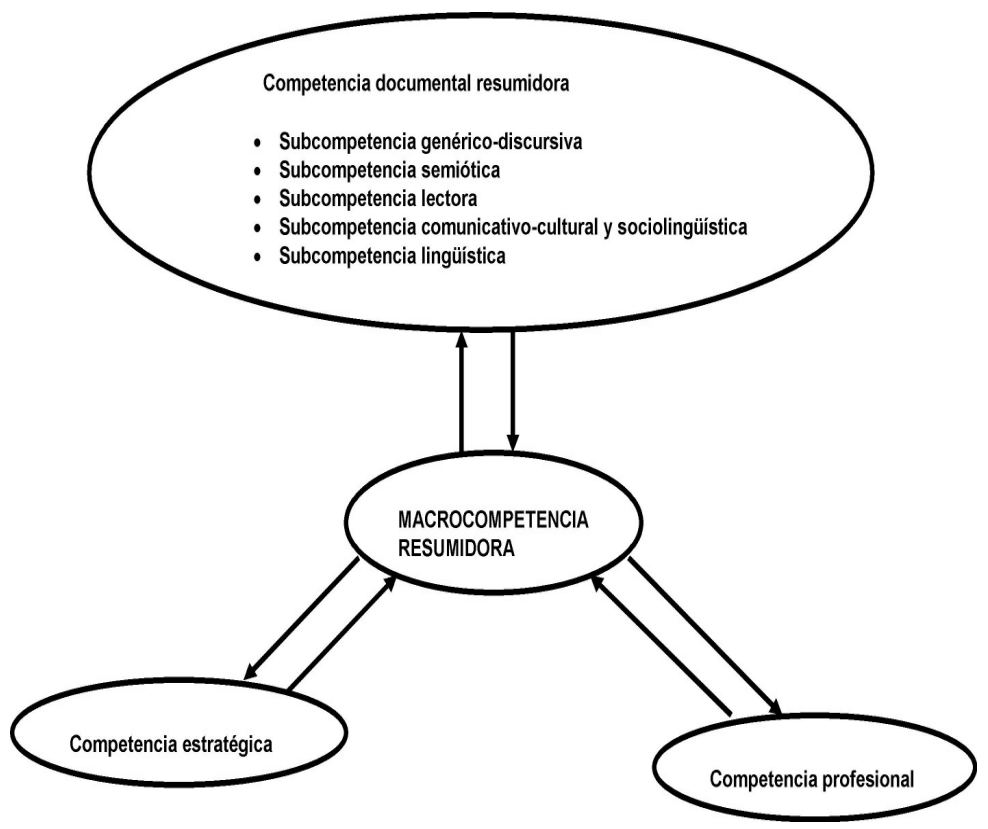

FIGURA 3 - Los componentes de la competencia resumidora Fonte: Elaboración propia.

Competencia documental-resumidora o meta-representativa (instrumental, técnica y tecnológica). Habilidad para realizar eficazmente el proceso de resumen documental de cualquier tipo textual con la generación de resúmenes que satisfagan las normas y convenciones de 
este género documental. Está integrada por las siguiente secuencia de subcompetencias:

Subcompetencia genérico-discursiva: habilidad para adecuar la estructura, contenido y estilo de un resumen al género discursivo y tipo de texto al que pertenece el objeto/texto a resumir. El género se presenta así como el recipiente en el que convergen todos los aspectos relevantes útiles para el análisis del proceso resumidor. El estudiante debe ser capaz, en primer lugar, de reconocerlo como categoría para, posteriormente, ir descendiendo a los niveles de análisis macro y microestructural que conformarán su competencia de género.

Subcompetencia semiótica: ante la integración interactiva de distintos canales y sistemas (textual, gráfico, sonoro, audiovisual, hipermedia, etc.) se necesitan habilidades para el reconocimiento y representación de los diferentes códigos [competencia textual, visual, audiovisual, sonora, mixta], así como de una competencia digital en entornos multimedia.

Subcompetencia lectora: relacionada con la competencia genérica, competencia de dominio temático, competencia extralingüística y competencia socio-retórica o cultural.

Subcompetencia comunicativo-cultural y sociolingüística: capacidad de interpretar y expresarse de acuerdo con el contexto, considerando: textos en otras lenguas, características de los estilos epistemológicos, retórica argumentativa, fraseología y terminología, etc.

Subcompetencia linguística: semántica, gramática textual, sintáctica y pragmática.

Competencia estratégica: dominio de las estrategias comunicativas y resumidoras que permiten compensar carencias 0 lagunas derivadas de dificultades específicas. Este tipo de competencia, junto con las habilidades metacognitivas, nos permitirá llevar a cabo el proceso general del resumen. Al mismo tiempo nos ayudará a construir expectativas adecuadas, a partir de situaciones específicas en un contexto de realización de un determinado tipo de resumen. Este tipo de subcompetencia dirige la aplicación de todas las demás y comprende todos aquellos procedimientos que se aplican a la organización y realización del trabajo resumidor, a la identificación y resolución de problemas, a la autoevaluación del proceso y a la evaluación de la calidad del resumen.

Competencia profesional (cultura de la profesión): conocimiento de las destrezas y habilidades asociadas a la práctica del tratamiento documental de contenido y a la ética profesional en la Información y Documentación. 
La macrocompetencia resumidora (MCR) es el sistema subyacente de conocimientos, habilidades y aptitudes necesarias para llevar a cabo el proceso resumidor en cada una de sus actividades. Como todo conocimiento experto, tiene componentes declarativos, procedimentales y actitudinales. Está formada por un conjunto de subcompetencias (documental, estratégica y profesional), entre las que existen interrelaciones, jerarquías y variaciones, que han de ser validadas durante los procesos de adquisición de dicha macrocompetencia. Las interacciones entre las distintas subcompetencias no se producen de un modo lineal y se van compensando y reestructurando, según va teniendo lugar el proceso de aprendizaje de las mismas.

Hemos de destacar también que la adquisición de la competencia resumidora es un fenómeno dinámico y que ésta se adquiriere progresivamente. El alumno ha de ir desarrollando un historial de competencia resumidora, trabajando una serie de habilidades adaptadas a las distintas formas y funciones del resumen documental, definiéndose así lo que convendremos en denominar espacio de construcción del resumen documental (fig.4), en el que la macrocompetencia global y las competencias locales resumidoras se irán asimilando significativamente por el alumno, en función de la ejercitación y la experiencia. En este contexto es muy importante la diversidad de situaciones comunicativas, el diseño instructivo (naturaleza, planificación y secuenciación de tareas), y la definición de los procesos de intervención del profesor como mediador del aprendizaje. El alumno ha de abordar una amplia variedad de tareas, en grado creciente de complejidad, en las que se ha de enfrentar a diferentes espacios-problemas ${ }^{7}$ (semánticos, retóricos, normativodocumentales, etc.). Este modelo didáctico supone el desarrollo de esquemas de conocimiento (cognitivos, procesuales, sociales, estratégicos y emocionales) que han de ser transformados, conscientemente, para que el alumno pueda reestructurar su conocimiento resumidor desde la adquisición de competencias metacognitivas. El alumno irá siendo cada vez más consciente sobre el proceso resumidor e ira adquiriendo un mayor bagaje metacognitivo sobre la propia actividad. Iniciar al alumno en todas estas competencias supone hacerle asumir los principios fundamentales que rigen el proceso resumidor y el propio concepto de resumen. Implica también enfrentarle a las dificultades que conlleva recorrer correctamente la actividad resumidora, preparándole para un resumen en contextos especializados donde abordará los problemas específicos de cada ámbito disciplinar y profesional.

\footnotetext{
Sobre el concepto de modelo cognitivo con estudio de procesos asociados, tomando como núcleo del aprendizaje la noción de problema y el desarrollo de estrategias Vid. Bereiter \& Scardamalia (1987, pp. 6-7). Los autores distinguen dos modelos complementarios en la adquisición de conocimiento (knowledge-telling model) y el de transformación-integración (knowledge- transforming model). Consideran asimismo un espacio de problemas de contenido y otro de problemas retóricos dentro de los entornos de tarea.
} 


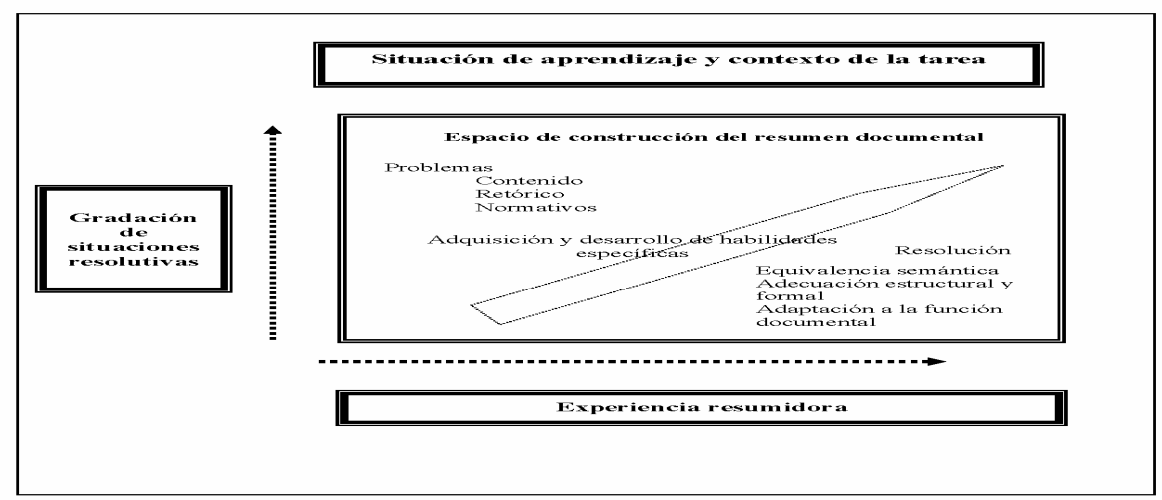

FIGURA 4 - Evolución de la competencia resumidora Fonte: Elaboración propia.

\section{Objetivos de aprendizaje del resumen documental}

La organización por objetivos de aprendizaje, junto con el diseño de propuestas metodológicas, supone un paso más en la didáctica y el desarrollo del currículum del resumen documental. Marcan la andadura didáctica del resumen e indican los resultados a los que debe conducir un proceso de aprendizaje en el marco de un programa de estudios.

El diseño del curriculum del resumen por objetivos de aprendizaje:

Permite diseñar la enseñanza-aprendizaje del resumen documental de manera sistemática y coherente.

Favorece la comunicación entre alumnos y profesores.

Facilita la elección de metodologías docentes.

Suscita la diversificación de las actividades pedagógicas.

Proporciona una base para la evaluación de los aprendizajes.

En los distintos programas docentes de las asignaturas relacionadas con el resumen contamos con relaciones más o menos detalladas de objetivos, expresados como resultados de aprendizaje y adquisición de competencias. No obstante, no todos atienden a una categorización y progresión adecuada que presuponga el ejercicio y el logro de dichas competencias. Así, se formulan objetivos orientados hacia el desarrollo de determinadas habilidades cognitivas y procedimentales, relacionadas con 
los principios básicos sobre los que se asienta el resumen documental y la actividad resumidora. En general, se orientan hacia la mejora de la competencia resumidora global en las distintas etapas del proceso resumidor: lectura, análisis y síntesis documental así como en la de expresión o escritura del resumen.

Expondremos una relación interdependiente e interactiva de objetivos generales (metodológicos, discursivo-textuales, estratégicos y de cultura profesional), desglosados a su vez en objetivos específicos. Hemos de señalar que este tipo de objetivos deberían proyectarse en diferentes niveles, en función de la fase del proceso de aprendizaje de que se tratase (iniciación o profundización). La adquisición adecuada de cada uno de ellos dependerá de la orientación metodológica adoptada en el aula y del diseño específico de tareas. El proceso de adquisición de la competencia resumidora es dinámico y en espiral. Así pues, es fundamental seguir una gradación en la consecución de los distintos objetivos conjugando éstos con las destrezas necesarias.

1.- Objetivos metodológicos: En primer lugar el alumno ha de captar los principios fundamentales sobre los que se asienta el proceso documental resumidor para lograr un correcto desarrollo del mismo y una equivalencia o adecuación semántico-funcional del resumen que representa al documento original. Sin embargo, dado el dinamismo de los procesos sociocognitivos implicados en las tareas de lectura y redacción del resumen, no existen soluciones únicas y tampoco se trata de crear automatismos fijos, puesto que los géneros textuales están en constante evolución. Ello ha de tener su reflejo tanto en el producto, el resumen documental, como en los procesos resumidores, desde técnicas de tratamiento y representación específicas.

\section{1.- El resumen documental como acto de comunicación social.}

Se trata de que el alumno capte la naturaleza y finalidad comunicativa desde una perspectiva social del resumen $y$, en consecuencia, la doble función del resumidor (receptor y generador) así como la importancia del usuario, destinatario final del resumen, como integrante de una comunidad discursiva determinada y de la finalidad de los resúmenes según el contexto comunicativo-documental y el entorno específico de realización del proyecto resumidor.

1.2. - La importancia de la aproximación previa a las características semiótico-discursivas de los originales a resumir.

El alumno ha de ser consciente de la variedad de dominios y ámbitos discursivos objeto de un posible resumen, aplicando técnicas documentales. Ha de sensibilizarse ante la necesidad de una observación cuidadosa y atenta de cada una de sus características esenciales de esos discursos como fase previa de planificación del proceso resumidor. 
1.3.- El interés de la fase de reconocimiento o lectura documental como paso previo para la representación y elaboración del resumen.

El alumno ha de asumir que ha de arbitrar técnicas selectivas de lectura. Ha de realizar una lectura activa, heurística y orientada, desde esquemas previos retórico-formales y de contenido. Asimismo, el estudiante ha de tener muy presente: a) los objetivos que han de guiar esta lectura documental: reconocer espacios formales de contenido en los que identificar y extraer información relevante y b) identificar las posibles zonas del documento en las que, dependiendo del tipo de material, se concentre el contenido con una mayor densidad temática. Queda claro que se realizará una lectura pasiva desde el punto de vista de la comprensión y el aprendizaje de textos, que requieren de otro tipo de técnicas lectoras y de estrategias de organización, representación y asimilación de la información.

1.4.- La trascendencia de la organización textual en relación con la búsqueda de la equivalencia semántica y la adecuación funcional del resumen.

El estudiante ha de asimilar el carácter contextual y dinámico de la representación documental en el resumen. El proceso de condensación o transubstanciación documental no es una simple síntesis de los elementos de significado del documento original sino un complejo proceso heurístico y funcional de equivalencias semánticas y adecuaciones pragmáticas materializadas en un nuevo documento, con autonomía propia y convenciones formales, semánticas y estilísticas.

1.5.- La importancia de los conocimientos extralingüísticos y la mirada hacia el usuario de información.

Este objetivo específico está relacionado con el anterior que profundizaba en la importancia del contexto textual. Ahora, la atención se dirige hacia los conocimientos extralingüísticos necesarios para la elaboración del resumen: conocimiento del entorno de la tarea a realizar, de los usuarios, del contexto del sistema de información y servicio resumidor en el que se realizar el proyecto resumidor, etc.

1.6.- La necesidad de desarrollar un espíritu crítico y reflexivo ante todo tipo de soluciones resumidoras.

Es necesario que nuestros alumnos desarrollen una competencia estratégica y reflexiva sobre su propio hacer, de modo que se sensibilice ante el error, aprendiendo a detectarlo y solventarlo adecuadamente. Asimismo, es importante también que se responsabilice de su producto y sepa defender ante los demás la idoneidad y adecuación del resumen elaborado y sepa dar soluciones alternativas en un proceso de revisión o evaluación de resúmenes. Este proceso reflexivo garantizará una mayor concentración e independencia en el proceso de elaboración de resúmenes en el aula y dará a los estudiantes una mayor seguridad en cuanto a las 
soluciones resumidoras adoptadas, enfrentándose a procesos de revisión explícitos con instrumentos como guías o plantillas de verificación.

\section{2.- Objetivos discursivos-textuales: géneros y tipologías textuales}

Este tipo de objetivos tratan de fomentar la competencia discursiva del resumidor de modo que éste se enfrente a diferentes espacios semióticos para que capte los diferentes problemas que afectan a la naturaleza del mensaje informativo; desde el punto de vista del canal y código utilizado, de su organización retórica y de la estructuración y presentación de los contenidos. Canal, código, estructura retórica y tipo de contenido son los distintos elementos que determinan que la información en un texto se organice de una u otra manera, que se den unos contenidos u otros, respetando el principio de adecuación a la función a la que sirven todos los textos-discursos. El alumno ha de ser consciente de que ha de aprender a mirar los textos que tiene que resumir, a bucear en sus convenciones discursivas (comunicativas, semióticas y pragmáticas), ya que cada género y tipo textual requieren de técnicas y estrategias resumidoras específicas. Cada uno de ellos generará también problemas de resumen diferentes y soluciones que habrán de tomarse desde el conocimiento de estos funcionamientos discursivotextuales.

2.1.- La diversidad de problemas en el resumen según los diferentes canales, géneros y tipos textuales.

Con este objetivo el estudiante ha de captar la importancia de los canales en los que circula y presenta la información, con especial atención en el canal digital como elemento innovador. El alumno ha de ver cómo afecta esta cuestión a la elaboración y evaluación de resúmenes en entornos digitales y su influencia en los nuevos comportamientos de los generadores y consumidores de información electrónica. Por otra parte, es asimismo relevante para el aprendizaje de los alumnos que éstos se enfrenten a las distintas problemáticas resumidoras que surgen al utilizar otros tipos de códigos semióticos no verbales en los documentos objeto de resumen (sonoro, audiovisual, gráfico, etc.) y cómo han de adaptarse a un código verbal para la representación del resumen documental. El aspecto de las restricciones de género y de los diversos tipos textuales dentro de éstos no es menos importante, como hemos señalado ya con anterioridad, así como la cuestión del análisis de dominio en cada uno de los géneros científicos y/ o profesionales. Incluimos aquí una problemática de "focos pragmáticos" difícil de solucionar puesto que no tiene su reflejo adecuado en la normativa sobre resumen documental. Surgen así problemas relacionados con los distintos campos temáticos, planteados por la complejidad informativa del documento, por la importancia de los elementos terminológicos; problemas de representación de modos textuales en los distintos ámbitos documentales (documentación audiovisual, publicitaria, jurídica, administrativa, científico-técnica, etc.) y su subordinación a códigos lingüísticos verbales, etc. 
2.2.- Necesidad de analizar las distintas convenciones retórico estilísticas de los distintos géneros y tipos textuales y trasladarlas a formatos 0 estructuras de resumen adecuadas para mantener la funcionalidad $y$ adecuación respecto al original $y$, al mismo tiempo, normalizadas para lograr una homogeneidad en los formatos de los resúmenes y un control en la representación y recuperación de información a través del resumen como herramienta documental y punto de acceso temático al documento original.

3.- Objetivos estratégicos o dominio de estrategias fundamentales en el proceso resumidor

3.1.- Aproximación a las metaestrategias de planificación y evaluación del proceso general resumidor que ponen en juego la competencia resumidora en todos los contextos de uso. Este tipo de objetivos se enfocan hacia la planificación, el control y la evaluación.

3.2.- Conocimiento de las estrategias locales resumidoras que ayudan a identificar y solucionar los problemas detectados en cada una de las fases del proceso resumidor en un proyecto concreto de resumen: estrategias de reconocimiento o lectura documental, estrategias de análisis-síntesis de la información, estrategias en la redacción del resumen, estrategias de revisión o corrección de resúmenes.

4.- Objetivos de cultura profesional este tipo de objetivos generales se enfocan hacia la asimilación de los fundamentos éticos y estilos de trabajo de los profesionales de la información y documentación en tareas de representación documental, enfrentando al alumno ante las diferentes "situaciones" resumidoras ante las que se puede encontrar.

4.1.- La necesidad de una aproximación real a los estilos de trabajo de los profesionales resumidores $y$ al resumen documental en sus diferentes ámbitos. Este objetivo específico plantea para que el alumno capte los fundamentos de los estilos de trabajo de los resumidores y de los profesionales de la documentación en contextos reales de actuación, ya que el entorno del sistema de información es uno de los factores que influye y determina los procesos de representación documental. Los alumnos habrán de aproximarse a las políticas resumidoras de los distintos servicios de información desde la revisión de los manuales de procedimiento resumidor, pautas y guías para la redacción de resúmenes o el análisis de los resúmenes difundidos en las distintas bases de datos de resúmenes. Complementariamente, el alumno puede obtener una visión más real del mercado del resumen en distintos ámbitos y contextos, así como de los diferentes productos o resúmenes en cada servicio resumidor. 
4.2.- Reflexión sobre la ética en los procesos de representación documental y el cumplimiento del compromiso social de los valores democráticos que han de regir todas las actividades de selección, organización, preservación difusión de la información. Ello nos lleva a una necesidad de políticas resumidoras explicitadas en códigos profesionales, manuales de procedimientos y normativas actualizadas, así como al análisis de la responsabilidad individual respecto al desempeño del rol de cada profesional en su actividad.

\section{Estrategias de aprendizaje en el resumen documental}

En los estudios pedagógicos y psicológicos, el término estrategia (del griego, strateguía, arte de dirigir las operaciones militares) designa la planificación conjunta de las directrices que se han de seguir en cada una de las fases de un proceso. Así entendida, consideramos las estrategias en cada una de las fases del proceso resumidor, como modelos de decisiones, y las relacionamos con las competencias y objetivos a desarrollar en cada una de ellas hasta lograr el resumen como meta final y resultado de la actividad global resumidora. Sirven como modelo de decisiones para lograr ciertos objetivos y su uso consciente amplía la capacidad y la eficacia del aprendizaje.

\section{QUADRO 1}

Las estrategias de aprendizaje en el resumen documental

Relación de estrategias sistémicas para el proceso de resumen documental

\begin{tabular}{|c|c|}
\hline Estrategias pre-resumidoras & $\begin{array}{l}\text { Planificación de la tarea resumidora } \\
\quad-\quad \text { Analizar la tarea } \\
-\quad \text { Identificar la tarea. } \\
-\quad \text { Establecer una meta. } \\
-\quad \text { Determinar y formular los pasos necesarios para } \\
\quad \text { cumplir la tarea. } \\
-\quad \text { Activar/ acceder a conocimientos previos. } \\
\text { Analizar factores ambientales. Contexto situacional o } \\
\text { interactivo del acto comunicativo del resumen documental } \\
\text {-Seleccionar estrategias adecuadas en relación con el } \\
\text { entorno. }\end{array}$ \\
\hline & $\begin{array}{l}\text { Estrategias para el reconocimiento documental: lectura } \\
\text { - Planificación de la tarea lectora } \\
\text { - Identificación de los objetivos de lectura } \\
\text { - Revisión de factores contextuales } \\
\text { - Determinación de expectativas de género: Reconocer } \\
\text { - el género y el tipo textual } \\
\text { Precisión de expectativas retóricas o de estructura- } \\
\text { formal: analizar y reconocer las estructuras de los }\end{array}$ \\
\hline
\end{tabular}




\begin{tabular}{|c|c|}
\hline \multirow[t]{5}{*}{$\begin{array}{l}\text { Estrategias según el tipo de } \\
\text { manipulación semiótico-textual }\end{array}$} & $\begin{array}{l}\text { textos } \\
\text { - Observación e identificación de marcadores } \\
\text { paratextuales (tablas, gráficos, figuras, et); } \\
\text { metatextuales e intertextuales }\end{array}$ \\
\hline & $\begin{array}{l}\text { Realización de una lectura rápida global (skimmig) para } \\
\text { obtener una compresión rápida del texto: temática principal y } \\
\text { estructuración formal: moves y tops o fases. } \\
\text { - Elaboración de una lectura de scanning detenida } \\
\text { entre los distintos moves: seleccionando y centrando } \\
\text { la atención en la información relevante del autor del } \\
\text { texto. } \\
\text { - Identificación y marcación de terminología } \\
\text { especializada o específica } \\
\text { Establecimiento de relaciones semánticas y de } \\
\text { relevancia entre distintos niveles: moves, contenidos } \\
\text { temáticos en cada move-submoves, conceptos y } \\
\text { términos. }\end{array}$ \\
\hline & $\begin{array}{l}\text { Estrategias de representación documental } \\
\text { - análisis-deconstrucción textual: Fijar expectativas } \\
\text { temáticas: identificación de la progresión formal y } \\
\text { temática (macrocohesión y macrocoherencia) y } \\
\text { organización de la información (Cohesión y } \\
\text { coherencia entre moves y submoves): selección y } \\
\text { extracción de la información más relevantes dentro } \\
\text { de cada move según la función de éste ; establecer } \\
\text { relaciones formales y conceptuales } \\
\text { síntesis-reformulación: condensación y } \\
\text { organización de la información según las } \\
\text { restricciones de género y normativa documental. } \\
\text { reexpresión- generación del resumen documental: } \\
\text { planificación y redacción-composición, acomodando } \\
\text { el texto a las convenciones del resumen documental } \\
\text { y a la función y tipo de resumen }\end{array}$ \\
\hline & Estrategias de revisión textual \\
\hline & $\begin{array}{l}\text { - Análisis de la adecuación del resumen al texto } \\
\text { original } \\
\text { - Cumplimiento de normas gramaticales y principios } \\
\text { de textualidad } \\
\text { - Correspondencia con la función y convenciones del } \\
\text { tipo de resumen a realizar. }\end{array}$ \\
\hline Estrategias de revisión global & $\begin{array}{l}\text { Revisión del proceso y del producto generado : supervisar, } \\
\text { verificar o corregir planteamientos y/ acciones } \\
\text { Revisión de estrategias cognitivas y metacognitivas } \\
\text { Reconsideración de estrategias personales }\end{array}$ \\
\hline
\end{tabular}




\section{Reflexiones finales}

A lo largo de este trabajo hemos subrayado la importancia de la didáctica en el resumen documental como otra de las dimensiones que ha orientar las investigaciones en esta operación de representación documental, subrayando la importancia de las competencias como uno de los conceptos clave para la enseñanza-aprendizaje del resumen y diseñando un modelo propio para la macrocompetencia resumidora. Asimismo, nos hemos ocupado de otros dos de los principios fundamentales que rigen la didáctica del resumen: los objetivos de aprendizaje y las estrategias. De este modo:

Se ha destacado la necesidad de que la didáctica del resumen documental tenga un espacio propio de reflexión e investigación, y sus resultados un reflejo tanto en los manuales sobre resumen, como en los distintos programas docentes que cubren los principios, técnicas y estrategias del resumen en cada uno de los diseños curriculares de los planes de estudio en Biblioteconomía y Documentación.

Se han expuesto, aunque sea de forma muy sucinta, los fundamentos básicos y operativos de los las principales características de la competencia resumidora, desde una aproximación a su naturaleza y componentes. Se ha ofrecido, así, un modelo para su funcionamiento, fundamentado en la observación de la actuación resumidora. No obstante, nos encontramos actualmente analizando la interrelación y jerarquía entre los componentes de dicho modelo, y diseñando un estudio para revisar y validar la evolución de los procesos de aprendizaje de dicha competencia, desde una revisión de las diferentes estrategias que interactúan en la dimensión operativa del proceso resumidor. El objetivo último sería intentar comprender e interpretar lo que sucede en nuestras aulas para poder transformar nuestra práctica resumidora desde una reflexión consciente y situada. Este tipo de investigación, realizada "desde" el aula y no "para" el aula, obliga a redefinir las fronteras entre investigación y aplicación, y facilita que las relaciones entre teoría y práctica no sólo sean constantes sino que se requieran y retroalimenten mutuamente.

Otra de las metas que nos hemos marcado era reflexionar sobre la formación competencial, en torno a la que se están diseñando los actuales planes de estudio de los grados en Información y Documentación, y sus implicaciones educativas aplicadas al resumen documental. La competencia resumidora es una noción compleja y su operativización requiere de un diseño adecuado de objetivos y estrategias para lograr ese nivel de desempeño competencial requerido, además de la adopción de metodologías didácticas adecuadas, centradas en las características propias del proceso resumidor y su aprendizaje consciente. Junto a la elección de una u otra metodología, para el logro de determinadas destrezas se ha de diseñar un marco de actividades que justifiquen el 
ejercicio y la adquisición de las competencias a nivel local y global. Sólo en base a estos niveles progresivos de representación consciente de las distintas actividades que conlleva el proceso resumidor será posible una reformulación y reestructuración de las mismas, y una supervisión adecuada por parte del profesor. Así, no se trata tanto de enseñar procedimientos y técnicas sino, fundamentalmente, de cambiar la percepción que los estudiantes tienen sobre el resumen documental y la actividad resumidora. Y ello, incidiendo en la asunción de concepciones más elaboradas que permitan poner en marcha procesos de representación documental más complejos que, finalmente, van a incidir en el aumento de la función epistémica y operativa del resumen documental.

\section{BIBLIOGRAFÍA}

BEREITER, C.; SCARDAMALIA, M. The psychology of written composition. Hillsdale, NJ: Lawrence Erlbaum, 1987.

CASSANY, D. Describir el escribir: como se aprende a escribir. Barcelona: Paidós, 1998.

COLLINS, A.; GENTNER, D. A framework for cognitive theory of writing: cognitive processes in writing. Hillsdale, NJ: Lawrence Earlbaum,1980.

CHOMSKY, N. Aspects of the theory of syntax. Cambridge: MIT Press, 1965.

EUROPEAN COUNCIL OF INFORMATION ASSOCIATIONS (ECIA). Euroreferencial en Información $y$ Documentación. Volumen 1: Competencias y aptitudes de los profesionales europeos de información y documentación. Madrid: SEDIC, 2004. Disponible en: <http:// www.certidoc.net>. Consultado el: 20 ago. 2009.

FLOWER, L.; HAYES, J. The dynamics of composing: making plans and jugging constraints. En: GREEG, L; STEINBERG, E. (Eds.). Cognitive processes in writing. Hillsdale, NJ: Lawrence Erlbaum, 1980. p. 39-58.

GARCÍA MARCO, F. J. et al. Diseño y coordinación curricular de las asignaturas de tratamiento y recuperación de la información de la diplomatura de biblioteconomía y Documentación. Zaragoza: Prensas Universitarias de Zaragoza, 2006.

GRAVE, W.; KAPLAN, R.B. Theory and practice of writing: an applied linguistic perspective. Londres: Longman, 1996.

IZQUIERDO ALONSO, M. Nuevos enfoques en el estudio del tratamiento documental de contenido desde los presupuestos de las ciencias del lenguaje. Revista SCIRE: representación y organización del conocimiento, v. 6, n. 1, p. 43-163, 2000.

- La investigación en el resumen documental: dimensiones, enfoques y técnicas. En: BORGES, M. M.; CASADO, E. S. (CoordS.). A 
Ciência da Informação criadora de conhecimiento. Coimbra: University press, 2009, pp.65-76.

IZQUIERDO ALONSO, M.; MORENO FERNÁNDEZ, L .M. El resumen documental: un reto didáctico. Madrid: ANABAD, 2009.

Perspectives of the Studies on document abstracting: towards an integrated view models and theoretical approaches. Journal of Documentation, v. 66, n. 4, 2010. (En prensa).

JOHNSON, P. La evaluación de la comprensión lectora: Un enfoque cognitivo. Madrid: Visor, 1989.

MOREIRO GONZÁlEZ, J. A.; TEJADA ARTIGAS, C. Competencias profesionales en el área de la Ciencia de la Información. En: VALENTIM, M. Atuação Profissional na área de informação. São Paulo: Polis, 2004.

PINTO, M. El resumen documental: paradigmas, modelos y métodos. Madrid: Fundación Germán Sánchez Ruipérez, 2001.

PINTO, M. et.al. Aprendiendo a resumir: prontuario y resolución de casos. Gijón: Trea, 2005

SCARDAMALIA, M.; BEREITER, C. Dos modelos explicativos de los procesos de comprensión escrita. Infancia y Aprendizaje, n. 58, p. 43-64, 1992.

Título de grado en Información y Documentación. Madrid: Agencia Nacional de Evaluación de la Calidad y Acreditación, 2004. Versión electrónica: <http://www.aneca.es/modal eval/docs/conver biblio.pdf>. Consultado el: 20 ago. 2009.

TEJADA ARTIGAS, C. et al.El diseño del plan docente en Información y Documentación acorde con el Espacio Europeo de Educación Superior: un enfoque por competencias. Madrid: Universidad Complutense, 2006.

TEJADA, C.; MEYRIAT, J. Competencias en información y documentación. Los proyectos europeos DECIDOC y CERTIDOC. Educación y Biblioteca, n. 137, p. 110-116, septiembre/octubre 2003.

WINOGRAD, P. Strategic difficulties in summarizing texts. Reading Research Quarterly, v. 19, n. 4, p.404-425, 1984. 